\title{
The Second ACM SIGEnergy Workshop on Reinforcement Learning for Energy Management in Buildings \& Cities (RLEM)
}

\author{
Zoltan Nagy \\ nagy@utexas.edu \\ The University of Texas at Austin \\ Jan Drgona \\ jan.drgona@pnnl.gov \\ Pacific Northwest National Laboratory
}

\author{
June Young Park \\ june.park@uta.edu \\ The University of Texas at Arlington \\ Matias Quintana \\ matias@u.nus.edu \\ National University of Singapore
}

\begin{abstract}
This is the second edition of the ACM SIGEnergy Workshop for Reinforcement Learning for Energy Management in Buildings and Cities (RLEM). RLEM brings together researchers and industry practitioners for the advancement of (deep) reinforcement learning (RL) in the built environment as it is applied for managing energy in civil infrastructure systems (energy, water, transportation).
\end{abstract}

\section{KEYWORDS}

reinforcement learning, energy

\section{ACM Reference Format:}

Zoltan Nagy, June Young Park, Jan Drgona, and Matias Quintana. 2021. The Second ACM SIGEnergy Workshop on Reinforcement Learning for Energy Management in Buildings \& Cities (RLEM). In The 8th ACM International Conference on Systems for Energy-Efficient Buildings, Cities, and Transportation (BuildSys '21), November 17-18, 2021, Coimbra, Portugal. ACM, New York, NY, USA, 2 pages. https://doi.org/10.1145/3486611.3491126

\section{INTRODUCTION}

Welcome to the second edition of RLEM, The Second ACM SIGEnergy Workshop on Reinforcement Learning for Energy Management in Buildings Cities! We are very excited to organize this workshop as part of BuildSys 2021. After an inspiring first edition RLEM20 with about 100 participants, we are looking forward to hear new developments in the field. The two main sessions this year focus on the two major challenges, Real-world implementations of RL algorithms in buildings and benchmarking of RL algorithms with other controllers. Worldwide experts will be presenting their latest research. In addition, at the request of the community we have a Testing Environment session with three environments from occupant scale, to building scale to urban scale for those who want to know how to get started in this field.

The COVID-19 pandemic forces us, again, to hold this event virtually, limiting the advantages of in-person discussions at the talks, the coffee breaks and at dinners. However, it provides us with the unique opportunity to welcome a large number of participants

Permission to make digital or hard copies of part or all of this work for personal or classroom use is granted without fee provided that copies are not made or distributed for profit or commercial advantage and that copies bear this notice and the full citation on the first page. Copyrights for third-party components of this work must be honored

For all other uses, contact the owner/author(s).

BuildSys '21, November 17-18, 2021, Coimbra, Portugal

(c) 2021 Copyright held by the owner/author(s).

ACM ISBN 978-1-4503-9114-6/21/11.

https://doi.org/10.1145/3486611.3491126 from all over the world, who otherwise may not have joined. We are excited and are looking forward to great discussions!

\section{CALL FOR PAPERS}

Buildings account for $40 \%$ of the global energy consumption and $30 \%$ of the associated greenhouse gas emissions, while also offering a $50-90 \%$ CO2 mitigation potential. The transportation sector is responsible for an additional $30 \%$. Optimal decarbonization requires electrification of end-uses and concomitant decarbonization of electricity supply, efficient use of electricity for lighting, space heating, cooling and ventilation (HVAC), and domestic hot water generation, and upgrade of the thermal properties of buildings. A major driver for decarbonization are integration of renewable energy systems (RES) into the grid, and photovoltaics (PV) and solar-thermal collectors as well as thermal and electric storage into residential and commercial buildings. Electric vehicles (EVs), with their storage capacity and inherent connectivity, hold a great potential for integration with buildings.

The integration of these technologies must be done carefully to unlock their full potential. Artificial intelligence is regarded as a possible pathway to orchestrate these complexities of Smart Cities. In particular, (deep) reinforcement learning algorithms have seen an increased interest and have demonstrated human expert level performance in other domains, e.g., computer games. Research in the building and cities domain has been fragmented and with focus on different problems and using a variety of frameworks. The purpose of this Workshop is to build a growing community around this exciting topic, provide a platform for discussion for future research direction, and share common frameworks. Topics of Interest

Topics of interest include, but are not limited to:

- Challenges and Opportunities for RL in Building and Cities

- Explorations of model vs model-free RL algorithms and hybrids

- Comparisons of RL algorithms to other control solutions, e.g., model-predictive control

- Frameworks and datasets for benchmarking algorithms

- Theoretical contributions to the RL field brought about by constraints/challenges in the buildings/cities domain

- Applications (demand response, HVAC control, occupant integration, traffic scheduling, EV/battery charging, DER integration) 


\section{REVIEW PROCESS}

As for the program and review process, for the total of 10 submitted papers the TPC chairs assigned three reviewers for each paper based on reviewers' expertise, preferences, and potential conflict of interest. With the comments of the reviewers, the TPC chairs discussed each paper thoroughly and considered their relevance to the community. Finally, the TPC chairs accepted 6 papers, and grouped them into two high quality sessions in the workshops. We are especially excited to have contributions from academia, national labs and industry.

\section{WORKSHOP PROGRAM}

Table 1: Workshop Program Schedule (tentative)

\begin{tabular}{|c|c|}
\hline Time (local) & Presentation \\
\hline $15: 00-15: 20$ & $\begin{array}{l}\text { Opening remarks, by General Chair and TPC } \\
\text { Chairs }\end{array}$ \\
\hline $15: 20-16: 00$ & $\begin{array}{l}\text { 1st Keynote: Gregor Henze (University of Col- } \\
\text { orado Boulder) }\end{array}$ \\
\hline $16: 00-16: 50$ & $\begin{array}{l}\text { Session 1: Addressing challenges of applying RL } \\
\text { in real-world buildings }\end{array}$ \\
\hline $16: 50-17: 00$ & Break \\
\hline $17: 00-17: 40$ & $\begin{array}{l}\text { 2nd Keynote: Helia Zandi (Oak Ridge National } \\
\text { Laboratory) }\end{array}$ \\
\hline $17: 40-18: 00$ & Community Announcements \\
\hline $18: 00-18: 50$ & $\begin{array}{l}\text { Session 2: Benchmarking RL with other controls } \\
\text { including other RLs }\end{array}$ \\
\hline $18: 50-19: 00$ & Break \\
\hline $19: 00-20: 20$ & Session 3: Testing environment \\
\hline $20: 20-20: 30$ & Closing remarks \\
\hline
\end{tabular}

\section{ACCEPTED PAPERS}

Session 1 - Addressing challenges of applying $R L$ in real-world buildings:

- Avisek Naug (Vanderbilt University ), Marcos QuinonesGrueiro (Vanderbilt University), Gautam Biswas (Vanderbilt University ), Sensitivity and Robustness of End-to-end Data-Driven Approach for Building Performance Optimization

- Marco Biemann (TU Denmark), Xiufeng Liu (TU Denmark), Yifeng Zeng (Northumbria University), Lizhen Huang (Norwegian University of Science and Technology), Addressing partial observability in reinforcement learning for energy management

- Khoder Jneid (Université Grenoble Alpes, LIG), Stephane Ploix (Grenoble INP), Patrick Reignier (Université Grenoble Alpes, LIG), Pierre Jallon (eLichens), Deep Q-Network Boosted with External Knowledge for HVAC Control

Session 2 - Benchmarking $R L$ with other controls including other RLs:

- Kuldeep Kurte (ORNL), Kadir Amasyali (ORNL), Jeffrey Munk (ORNL), Helia Zandi (ORNL), Comparative Analysis of
Model-Free and Model-Based HVAC Control for Residential Demand Response

- Javier Jiménez-Raboso (Universidad de Granada), Alejandro Campoy-Nieves (Universidad de Granada), Antonio ManjavacasLucas (Universidad de Granada), Juan Gómez-Romero (Universidad de Granada), Miguel Molina-Solana (Universidad de Granada), Sinergym: A Building Simulation and Control Framework for Training Reinforcement Learning Agents

- Ruben Glatt (LLNL), Felipe Leno da Silva (LLNL), Braden Soper (LLNL), William A. Dawson (LLNL), Edward Rusu (LLNL), Ryan A. Goldhahn (LLNL), Collaborative energy demand response with decentralized actor and centralized critic

\section{ORGANIZATION}

General Chair

- Zoltan Nagy (University of Texas at Austin)

Technical Program Committee Co-Chairs

- June Young Park (University of Texas at Arlington)

- Jan Drgona (Pacific Northwest National Laboratory)

Web Chair

- Matias Quintana (National University of Singapore)

Technical Program Committee

- Anand Krishnan Prakash (Lawrence Berkeley National Lab)

- Anjukan Kathirgamanathan (University College Dublin)

- Ankush Chakrabarty (Mitsubishi Electric Research Laboratories)

- Bharathan Balaji (Amazon)

- Bratislav Svetozarevic (Swiss Federal Laboratories for Materials Science and Technology)

- Giuseppe Pinto (Politecnico di Torino)

- Hari Prasanna Das (University of California, Berkeley)

- Helia Zandi (Oak Ridge National Laboratory)

- Jose Vazquez-Canteli (Mapped)

- Kuldeep Kurte (Oak Ridge National Laboratory)

- Matias Quintana (National University of Singapore)

- Ming Jin (Virginia Tech)

- Omid Ardakanian (University of Alberta)

- Silvio Brandi (Politecnico di Torino)

- Xin Jin (National Renewable Energy Laboratory)

- Zhe Wang (Lawrence Berkeley National Lab)

- Zhiang Zhang (University of Nottingham Ningbo China)

\section{ACKNOWLEDGEMENT}

We would like to thank all the authors for their excellent submissions to RLEM'21, and also the technical program committee members for their hard work reviewing and critiquing the papers. Lastly we thank the BuildSys 2021 workshop chairs Bharathan Balaji and Sid Chi-Kin Chau as well as the general chair of Buildsys 2021, Xiaofan Jiang, for making RLEM'21 possible. 